\title{
Euronews, un laboratoire de la production de l'information « européenne »
}

Olivier Baisnée et Dominique Marchetti

\section{(2) OpenEdition \\ 1 Journals}

Édition électronique

URL : http://journals.openedition.org/conflits/283

DOI : $10.4000 /$ conflits.283

ISSN : $1777-5345$

Éditeur :

CCLS - Centre d'études sur les conflits lilberté et sécurité, L'Harmattan

Édition imprimée

Date de publication : 1 juin 2000

ISBN : 2-7384-9946-5

ISSN : 1157-996X

\section{Référence électronique}

Olivier Baisnée et Dominique Marchetti, «Euronews, un laboratoire de la production de l'information « européenne » », Cultures \& Conflits [En ligne], 38-39 | été-automne 2000, mis en ligne le 13 mars 2006, consulté le 30 mars 2021. URL : http://journals.openedition.org/conflits/283 ; DOI : https://doi.org/ $10.4000 /$ conflits. 283

Ce document a été généré automatiquement le 30 mars 2021.

Creative Commons License 


\title{
Euronews, un laboratoire de la production de l'information « européenne »
}

\author{
Olivier Baisnée et Dominique Marchetti
}

1 Cet article est le premier état d'une recherche monographique sur la chaîne d'information en continu Euronews visant à comprendre in vivo comment fonctionne ce qu'on appelle un média à vocation européenne, voire même transnationale, c'est-àdire qui traite d'informations internationales destinées à des publics situés dans des aires géographiques (l'Europe et le Bassin méditerranéen essentiellement) très diverses. Le cas d'Euronews, créée en 1992 et lancée le 1er janvier 1993, paraissait particulièrement pertinent pour étudier la production d'une information et d'un journalisme à prétention européenne dans la mesure où ses programmes sont diffusés en six langues (allemand, anglais, espagnol, français, italien et portugais) et fabriqués par des équipes de journalistes de nationalités différentes. Autrement dit, il s'agit de tenter d'apporter une contribution sociologique aux débats portant sur les questions de « construction européenne » à travers l'étude d'une sorte de « laboratoire » européen"1, la quasi-totalité du personnel de la chaîne (notamment la rédaction) étant concentrée en un seul lieu. A travers cet exemple très concret, on peut cerner comment des individus cherchent à leur façon à homogénéiser des points de vue sur l'information (« covering world news with one common vision $»^{2}$ ), des pratiques journalistiques, des hiérarchies de l'information très différentes, pour essayer de construire un contenu éditorial ou un journalisme "à l'européenne ». C'était également une manière de traiter ces questions à partir d'une expérience journalistique et non politique, ce qui est très rarement le cas dans les recherches sur ce thème $e^{3}$. Parce que la chaîne rassemble des journalistes de diverses nationalités, elle constitue également un terrain propice à la collecte d'informations sur différents champs journalistiques nationaux d'autant plus que la démarche comparative est très peu utilisée dans les recherches sociologiques sur les médias ou le journalisme. Si la comparaison reste forcément très limitée dans le cadre de cette recherche, elle aura peut-être le mérite de dégager un certain nombre de pistes de réflexion. Ce sont ces deux aspects de l'enquête qui seront privilégiés ici ${ }^{4}$. 
Après avoir dressé un bref historique de la création de la chaîne en la replaçant dans les différents espaces (politique et médiatique) auxquels elle est liée, il s'agit d'abord de montrer quelles sont les contraintes qui pèsent sur ce média à prétention européenne, ce qui est une manière de s'interroger sur les obstacles à l'édification d'un média transnational. On analyse ensuite la manière dont les journalistes cherchent à construire « un point de vue européen » sur l'actualité dite internationale à travers leur travail et leur organisation au quotidien. Des espaces médiatiques et politiques en pleine transformation Comprendre la genèse et le développement de cette chaîne implique de la situer rapidement dans l'évolution des champs journalistique et politique nationaux en Europe et des relations qu'ils entretiennent entre eux ${ }^{5}$. La création et le développement d'Euronews s'inscrivent dans un contexte de transformations des espaces médiatiques nationaux, notamment pour les chaînes de télévision ${ }^{6}$. Au-delà de l'accroissement de l'offre qui s'est traduit par la multiplication du nombre de chaînes diffusées par voie hertzienne, le câble ou le satellite, ces espaces ont aussi été marqués par un mouvement de segmentation des publics et donc des publicités. A côté des chaînes généralistes existantes, se sont développées des chaînes spécialisées s'adressant à des publics plus restreints: des télévisions thématiques (musique, sport, information, voyage, cinéma, séries, religion, etc.) ou visant des publics situés en dehors du cadre géographique national traditionnel. D'un côté, des chaînes locales ou régionales ont été créées et se sont développées à des degrés divers suivant les pays, certaines chaînes à vocation nationale ou internationale ont «nationalisé » ou " régionalisé » leurs programmes en développant des décrochages, des publicités ou en multipliant les langues ${ }^{7}$; d'un autre côté, des chaînes transnationales ont vu le jour - notamment dans trois domaines majeurs dont les activités sont tout particulièrement internationalisées ${ }^{8}:$ la musique, l'information et le sport avec les exemples emblématiques de MTV (1981), CNN (1980), Eurosport (1989) et BBCWorld (1991) - et des chaînes généralistes nationales ont diffusé des versions étrangères ou leur version nationale à l'étranger. Des programmes portant sur l'Europe ont également été créés sur des chaînes nationales comme en France ou en Angleterre9. Des évolutions comparables pourraient être étudiées dans la presse écrite où les formules ont connu des résultats très inégaux. Une autre série de transformations des espaces médiatiques nationaux doit beaucoup à l'intensification de la concurrence commerciale, du fait de la multiplication de l'offre télévisuelle. Elle a provoqué des concentrations, avec la création d'importants groupes de communication ou l'arrivée de grandes entreprises dans le secteur des médias, une internationalisation des sociétés dont les marchés dépassent les cadres géographiques nationaux, et une diversification dans la mesure où ces entreprises ont des activités dans différents domaines et sont souvent multimédias. Si les situations sont très variables de pays à pays, ce renforcement de la concurrence a eu pour effet de développer ou de renforcer le pôle des chaînes privées dont le nombre est devenu supérieur aux chaînes publiques dans les pays d'Europe : il y avait quatre télévisions privées en Europe en 1982, elles étaient 58 en 1992 et plus de 250 en $1997^{10}$. La genèse et le développement d'Euronews doivent donc être resitués dans ce contexte du début des années 90 dans lequel les principaux membres de l'Union européenne de radio-télévision (UER), c'est-à-dire les chaînes publiques, cherchent notamment pour des raisons économiques à consolider leurs relations au sein de cette organisation dans le domaine de l'information et du sport ${ }^{11}$. "L'EBU a été forcée de reconsidérer son propre rôle : d'une organisation représentative de toutes les télévisions nationales européennes, elle est devenue la représentante d'un 
groupe d'intérêt, les télévisions publiques ", écrit ainsi Tony Naets, le responsable de la coordination Actualités de l'UER ${ }^{12}$. C'est ainsi qu'Eurosport est créée en 1989 sur l'initiative de l'UER tandis qu'Euronews est fondée en 1992 par onze membres actifs ${ }^{13}$ auxquels se sont ajoutées plus tard d'autres chaînes partenaires. Ce projet politico-économique n'est également pas sans rapport avec les transformations des espaces politiques nationaux compte tenu de l'importance des dimensions européennes des politiques publiques et des réglementations dans le domaine audiovisuel. En effet, la création d'Euronews s'inscrit dans le prolongement de deux projets de création de " chaîne européenne » qui se solderont par des échecs dans les années 80 : EURIKON, envisagée par la Commission européenne, et Europa TV, née d'une initiative néerlandaise. Euronews est également le produit d'un projet politique au sens large porté par certains dirigeants européens, notamment par le gouvernement français, à travers des directives communautaires ou des soutiens financiers. Il s'agit, au moins dans le discours, de lutter contre la domination américaine, voire anglo-saxonne ${ }^{14}$, sur l'information dite internationale (et plus largement sur la "culture " telle que les institutions européennes la définissent), surtout à la suite de la guerre du Golfe qui a mis en évidence la suprématie de CNN comme source d'images. Il s'agit aussi de renforcer les télévisions nationales de service public en Europe et de trouver un nouveau support ${ }^{15}$ participant à la construction d'une « identité européenne ». Dans un livre vert publié en $1984^{16}$, la Commission énonce les principes suivants : « l'information est un facteur décisif, peut être le plus décisif, de l'unification européenne... l'unification européenne sera seulement achevée si les Européens le veulent. Les Européens ne le voudront que s'il y a quelque chose comme une identité européenne. Une identité européenne ne se développera que si les Européens sont informés de manière adéquate. Jusqu'à présent l'information passant par les médias de masse est contrôlée au niveau national ». De son côté, un autre document de la Commission ${ }^{17}$ explique en 1985 : « une communauté de culture en Europe est déjà un fait indéniable. Au-delà de la diversité de surface des langues, goûts et styles artistiques, il y a une ressemblance, une similarité, une dimension ou une identité européenne basées sur un héritage culturel commun. Les contributions des différents individus, idées, styles et valeurs ont, à travers les siècles, créés notre civilisation commune». On voit bien apparaître dans le discours de la Commission sur les questions de culture et d'identité le volontarisme politique et la logique qui conduira à soutenir la création d'une chaîne comme Euronews. Le paradigme tel que le résume Philip Schlesinger est le suivant: "L'information (la culture) est ici présentée comme agissant comme un homogénéisateur ou un articulateur de la volonté: il s'agit d'une perception extrêmement idéaliste et volontariste de la construction de l'ordre social désiré, et une explication pour le moins improbable ${ }^{18}$. D'ailleurs dès 1985, le rapport Adonnino ${ }^{19}$ commandé en 1984 par le Conseil européen à la suite de la faible participation aux élections européennes suggérait, parmi d'autres mesures visant à "renforcer et promouvoir l'identité de la Communauté et son image à la fois pour ses citoyens et pour le reste du monde", que soit créée une "aire audiovisuelle» européenne passant notamment par la mise en place d'une chaîne télévisée plurilinguistique "véritablement européenne ». C'est cette logique qui se concrétisera dans la création d'Euronews dans la foulée de la ratification d'un traité de Maastricht mettant l'accent sur les questions de citoyenneté européenne. D'ailleurs, lorsqu'il s'interroge sur les moyens nécessaires à la bonne communication sur l'Union européenne ${ }^{20}$, le Parlement européen évoque explicitement Euronews comme un des vecteurs pouvant être utilisé : 
«Le Parlement et la Commission doivent promouvoir (...) la production d'émissions télévisées sur l'activité des institutions européennes, qui pourraient être diffusées par Euronews ou d'autres médias ». Plus loin, dans le même rapport, les parlementaires européens indiquent qu'ils souhaitent, comme la Commission de la culture, de la jeunesse, de l'éducation et des médias de cette assemblée l'a évoqué, que «la subvention accordée à Euronews soit remplacée par un contrat d'information en 1998 de manière à permettre à l'UE d'utiliser Euronews comme un instrument de sa politique d'information à part entière ». Ce changement dans la politique de soutien à une chaîne « aux exceptionnelles compétences multilingues » se traduira par la mise en place d'un système de coproduction d'émissions mettant en scène un certain nombre de politiques communautaires. On voit bien là à la fois la manière extrêmement volontariste dont est envisagée l'information comme moyen d'unification des identités et de faire émerger une véritable identité européenne et la conception de la chaîne qui prévaut parmi les parlementaires européens : celle d'un instrument de la communication de l'UE ${ }^{21}$. Même si dans le fonctionnement quotidien de la chaîne, Euronews n'est pas subordonnée aux institutions européennes, le discours que tiennent les acteurs politiques européens sur l'information en général et sur Euronews en particulier est un bon indicateur de l'intérêt accordé à ce projet rédactionnel. Des logiques politiques, économiques et médiatiques Il existe donc derrière la création de cette chaîne une forte volonté politique au niveau communautaire qui vise à favoriser la naissance d'un média multilingue à vocation européenne susceptible, selon eux, de favoriser l'émergence d'une identité véritablement européenne. Cependant, une fois la chaîne créée, celle-ci va se heurter à des réalités très concrètes. Les difficultés rencontrées par la chaîne dès son lancement témoignent des contraintes pesant sur un projet de « média européen ». Les conditions sociales et historiques de possibilité de la création et du développement d'Euronews ne peuvent se comprendre qu'en prenant en compte des logiques à la fois médiatiques, économiques et politiques. Ces trois dimensions traversent la courte histoire d'une chaîne passée, par un glissement progressif, d'une logique initiale de chaîne de service public à une logique de chaîne commerciale. Pour résumer, elle se décompose en deux phases entre 1993 et aujourd'hui. La première se caractérise par une certaine improvisation à la fois politique, économique et éditoriale entre 1993 et 1997. Si le lancement de la chaîne reste une expérience sans précédent pour les membres de la rédaction de l'époque qui y voient un projet éditorial passionnant et novateur, il va vite se heurter à des contingences à la fois politiques et économiques. La rédaction va être traversée par des luttes politiques entre les dirigeants nommés par les principales chaînes fondatrices et la volonté politique des initiateurs du projet n'est pas suivie des financements nécessaires. Non seulement les rentrées publicitaires (l'existence d'un marché pan-européen est problématique) ne sont pas à la hauteur des espérances mais les dirigeants de la chaîne se heurtent aussi et surtout à l'insuffisance des recettes publiques (chaînes partenaires, instances locales, Communauté européenne notamment). L'ouverture du capital de la société opératrice de la chaîne au secteur privé - plus précisément à la vente de $49 \%$ des parts à Alcatel-Alsthom l'entreprise française devenant à la fin mai 1995 le principal opérateur par le biais de sa filiale la Générale Occidentale, marque le primat (encore relatif) des logiques économiques sur les logiques politiques. Jusqu'au rachat des parts par le groupe britannique ITN en 1997, cette première période de l'histoire de la chaîne est aussi marquée par une grande improvisation éditoriale comme en témoignent plusieurs journalistes situés à des niveaux hiérarchiques différents. "On faisait du remplissage 
d'antenne (sourire ironique), on faisait un journal qui si tu veux... Le journal, on le faisait le matin, il était encore là le lendemain matin quoi (...) Il y avait une Canadienne qui bossait ici avant qui avait trouvé cette formule très drôle, c'est...Euronews, attends c'était quoi ? Yesterday news for Tomorrow, ça collait pas mal à l'époque (rires), c'était vraiment ça. » (Entretien avec une journaliste, mars 2000) «C'était une espèce de no man's land parce que, bon, il n'y avait pas de tête, il n'y avait pas de direction (...) En deux ans si vous voulez, on a changé trois fois d'habillage. Ce qui est déjà une absurdité en soi. Editorialement, c'était bon le flou artistique, chacun faisait ce qu'il voulait. Les chefs d'édition... enfin moi quand j'étais à la maison que je regardais Euronews, je pouvais reconnaitre d'après le journal, le contenu du journal et la forme, qui était chef d'édition. » (Entretien avec un rédacteur en chef adjoint, avril 2000) Le rachat des parts d'Alcatel-Alsthom, qui revend alors l'intégralité de son secteur médias, par ITN correspond à une deuxième phase confortant la logique commerciale de la chaîne et surtout sa relative professionnalisation. Le consortium britannique, détenu notamment par des grands groupes comme Reuters, Carlton Communication et Granada Group, qui fournit les journaux d'information de trois chaînes anglaises ITV, Channel 4, Channel 5 cherche à se placer sur le marché international en faisant d'Euronews sa " vitrine » face à son concurrent $\mathrm{BBC}$ World. Les dirigeants du groupe s'attachent à restructurer la chaîne pour rétablir une situation financière très difficile en cherchant à accroître les recettes publicitaires. De la même manière, il s'agit de « sauver la réputation de cette chaîne ${ }^{22}$ pour reprendre l'expression du directeur de la rédaction en changeant les programmes et l'habillage : renforcement des journaux au détriment des magazines, de certaines rubriques (Sport, Economie, etc.), priorité aux directs, développement d'une information de proximité, etc. L'Europe d'Euronews, une définition sous contrainte Audelà de ces obstacles, Euronews a dû aussi faire face dès ses débuts au défi de la définition de son objet, c'est-à-dire de l'identité d'une chaîne dite européenne. En sortant des cadres nationaux ou internationaux traditionnels d'exercice du travail journalistique, la chaîne a peu à peu défini ce que signifiait l'ambition de proposer une information à vocation européenne. Qu'il s'agisse de problèmes de langage journalistique pour s'adresser à de larges audiences distinctes de celles des chaînes nationales de télévision, ou d'établir des habitudes de travail en matière de sélection et de traitement de l'information, Euronews invente en permanence, sous l'effet d'un certain nombre de contraintes pesant sur son fonctionnement quotidien, une définition de «son » Europe. Comme l'indique Chris Shore à propos des rédacteurs des rapports des institutions européennes, «forger une 'identité européenne' supérieure était simplement une question de greffer une identité collective supérieure sur et au-dessus des identités nationales et régionales, comme autant de poupées russes ou de boîtes chinoises " ${ }^{23}$. Cependant, comme le fait remarquer Philip Schlesinger, "malgré les proclamations rhétoriques, l'UE ne possède pas de culture ni d'identité transcendantes et communes analogues aux cultures et aux identités nationales des Etats qui la composent ${ }^{24}$. Or, le traitement journalistique de l'information s'appuie en général sur des références socialement partagées, qu'il n'est pas besoin d'expliciter. Dans le cadre d'une chaîne dont le principe est de s'adresser à une audience multinationale qui, par définition et malgré le discours volontariste de la Commission, ne possède pas de tels référents communs, la construction du ou des point(s) de vue, rarement envisagée sous cet angle dans une chaîne nationale, devient alors problématique. Elle est contrainte de plusieurs manières. La dépendance vis à vis des agences audiovisuelles Comme toutes les chaînes de télévision qui font de l'information, tout particulièrement en continu, 
Euronews est très largement dépendante de différentes sources d'images. Elle l'est d'autant plus que, faute de moyens financiers suffisants, la quasi-totalité des images ${ }^{25}$ diffusées proviennent de sources externes. En effet, Euronews est une chaîne dite de post-production, c'est-à-dire une télévision sans caméras ou presque. Elle relaie des images venant d'ailleurs et ne dispose ni de présentateurs ni de plateaux avec des journalistes. Sur ces images identiques, les commentaires sont diffusés dans six langues différentes. Les principales sources audiovisuelles sont les deux agences d'images internationales APTN (Associated Press Television News) et Reuters TV (RTV) auxquelles elle est abonnée, les chaînes partenaires qui échangent leurs images dans le cadre de l'Union européenne de radio-télévision (UER) et Independent Television News (ITN), le principal actionnaire. L'un des rédacteurs en chef adjoint résume : «On est une agence d'information télévisée, on prend, on coupe et on recoupe et on livre quelque chose ${ }^{26}$. Cette dépendance contraint bien évidemment la définition de l'Europe donnée par la chaîne. Compte tenu de la volonté de proposer un point de vue européen sur l'actualité, les sources d'images, même multiples, se révèlent parfois inadéquates pour illustrer les sujets d'Euronews. Cette contrainte très forte introduit deux types d'effets. Premièrement, les choix éditoriaux des grandes agences anglo-saxonnes Reuters TV est britannique et APTN américaine - en situation de quasi-monopole sur les images de l'actualité internationale ont bien évidemment des effets plus ou moins directs sur ceux d'Euronews. De nombreux thèmes comme l'«emploi» sont particulièrement délicats à traiter comme l'indiquent les responsables des rubriques couvrant les questions économiques (Economia) et les politiques européennes (Europa), qui sont souvent amenés à mobiliser les archives et les ressources infographiques (cartes, graphiques, etc.), voire parfois obligés d'abandonner certains sujets, faute d'images. La dépendance de la chaîne à l'égard de ses fournisseurs anglo-saxons incite les journalistes à ne pas se limiter à l'Europe des traités communautaires stricto sensu et à traiter plus traditionnellement l'actualité internationale, y compris avec une hiérarchisation de l'information différente des concurrents. Il n'en demeure pas moins que ce quasi-monopole des agences anglo-saxonnes suscite régulièrement des débats internes. "On dépend des fournisseurs. Un exemple: je vois une dépêche très sympathique, très intéressante et tout ça. Si je n'ai pas les images, je ne peux pas faire ce sujet-là. Voilà. Et les images par rapport aux agences de... aux grandes agences, c'est pas toujours simple d'y travailler. Parce qu'ils ont leur vision à eux, tu dépends de la vision des autres. Et on dépend de la vision d'une culture qui parfois n'est pas nécessairement liée à la culture d'Euronews, n'est pas liée à la culture européenne, n'est pas liée à la culture de certains pays européens. On dépend en gros d'une mentalité et d'une culture anglo-saxonnes. Voilà. Et je trouve que le... tous les problèmes qu'il y a ici dérivent surtout de cette... Parfois on a les journalistes espagnols, italiens et tout ça qui se fâchent, qui disent 'hé, en Espagne, il s'est passé ça. Pourquoi on l'a pas fait. Par contre, on a fait... on a mis dans le journal euh... le chat de la dame anglaise qui était allé sur l'arbre, ils sont partis le sauver. Pourquoi l'Angleterre, pourquoi l'Amérique et pas l'Espagne, pas l'Amérique latine, pas l'Italie, pas...'. Mais la raison c'est que ce n'est pas un choix éditorial, c'est simplement une nécessité... concrète. » (Entretien avec un journaliste italien, responsable de rubrique, avril 2000) Le second effet de cette dépendance - plus minime - est que les images d'Euronews provenant des EVN sont "nationales " au sens où elles ont été prises à l'origine pour satisfaire des journaux nationaux. Il est complexe de comprendre et de montrer très précisément en quoi elles peuvent apparaître comme un point de vue 
«national " ${ }^{27}$ sur un événement sauf à comparer la couverture par des médias nationaux différents qui ont des équipes sur place. Mais tout laisse à penser que les conclusions d'Olivier Boyd-Barret et Michael Palmer dans leur livre sur les agences de presse mondiales restent valides. Ces deux auteurs avaient mis en évidence combien les bureaux locaux contribuaient à relayer «les définitions des informations journalistiques nationales au niveau international» et combien ces agences "présentent une vision du pays où dominent les informations recueillies dans la capitale et auprès des sources officielles $"{ }^{28}$. Une chaîne européenne...commerciale La dimension européenne d'Euronews ne doit pas faire oublier non plus qu'elle est aussi et peut-être avant tout une chaîne d'information en continu commerciale, c'est-à-dire deux caractéristiques qui pèsent fortement sur ses productions. Tout d'abord, ces contraintes économiques se manifestent à travers les publics (c'est-à-dire aussi les publicitaires parce que la chaîne est "vendue » deux fois) auxquels s'adressent la chaîne. L'importance des mesures d'audience se matérialise d'une manière différente de celle des chaînes hertziennes traditionnelles. Il n'y a pas des mesures aussi précises des programmes («On reste dans un fonctionnement très, très empirique », explique un rédacteur en chef adjoint ${ }^{29}$ ) même si le " prime time " d'Euronews se situe le matin entre $6 \mathrm{~h}$ et $10 \mathrm{~h} 30$ et le soir à partir de $19 \mathrm{~h}$. Les chiffres les plus importants pour le service commercial et le service de la distribution de la chaîne sont non seulement les mesures d'audience générale dans les principaux pays européens mais surtout les chiffres d'audience potentielle (le nombre de foyers qui reçoivent Euronews) qui sont calculés à partir de la distribution de la chaîne. En effet, à l'inverse des chaînes " payantes » du câble et du satellite, elle recherche moins les revenus des distributeurs - la chaîne leur est souvent proposée gratuitement - qu'une audience potentielle maximale. Selon les chiffres communiqués par la chaîne, elle touchait en 1999, 94 millions de foyers en Europe par voie hertzienne - ses programmes sont repris par des grandes chaînes généralistes nationales - le câble et le satellite où elle est très présente dans les «services de base" ${ }^{30}$. De plus en plus dépendantes des ressources publicitaires ${ }^{31}$, Euronews s'adresse premièrement à un public relativement ciblé comme l'exprime clairement un dépliant du service Communication diffusé en 2000: «une audience intelligente d'un haut niveau socio-culturel ». Deuxièmement, elle est à la recherche d'une distribution géographique maximale. C'est pourquoi, elle a besoin de dépasser le strict cadre communautaire dans sa distribution et dans son actionnariat. Comme plusieurs organisations européennes (l'UER dans l'audiovisuel ou l'UEFA dans le football), Euronews compte parmi ses membres (Chypre, Grèce, Égypte, Tunisie, Algérie, Slovénie, République Tchèque et Roumanie) et plus encore ses principaux pays diffuseurs de nombreux pays de l'Europe de l'Est et du bassin méditerranéen. Au-delà de ses principales zones de diffusion, ses dirigeants cherchent une distribution sur tous les continents. Ainsi, la mise en place récente de la langue portugaise s'inscrit dans un projet à plus long terme d'implantation plus forte en Amérique latine. Les contraintes économiques se manifestent également dans le positionnement éditorial affiché par la chaîne. Celui-ci s'explique aussi par le fait qu'elle a dû se constituer et doit conforter des positions au sein d'espaces médiatiques nationaux dans lesquels ses principaux concurrents (auprès du public et des distributeurs ${ }^{32}$ ) sont certes les chaînes nationales d'information en continu quand elle existe mais surtout CNN et BBC World. La vocation européenne lui permet donc d'occuper une "niche» sur ces marchés hautement concurrentiels, celle laissée libre par les grandes chaînes anglo-saxonnes d'information en continu et par les chaînes nationales qui accordent une place très inégale à 
l'information internationale dans leurs programmes d'information ${ }^{33}$. Ses dirigeants au moins dans les discours cherchent à se démarquer d'une conception trop anglosaxonne et/ou américaine de l'information internationale et à mettre en exergue l'information dite européenne. Le directeur de la rédaction explique la position que cherche à occuper la chaîne face à ses concurrents ${ }^{34}:$ «Il y a beaucoup, beaucoup de concurrence, CNN évidemment et BBC, mais aussi les chaînes nationales. Pour nous, contre cette grande concurrence, il est très important je crois de trouver une position unique dans le marché. Et pour nous, la position unique est : nous ne sommes pas une chaîne nationale mais également nous ne sommes pas une chaîne globale comme CNN ou la BBC. Nous sommes une chaîne européenne (...) Et aussi nous sommes uniques au niveau des langues. Le fait que nous parlons aux publics dans leur propre langue. La BBC... CNN un peu avec CNN turque, ils commencent des accords avec des chaînes locales. Mais au niveau de toutes les heures de la journée, avec un agenda toujours européen, nous sommes uniques. Des exemples... Par exemple où Bill clinton a livré le 'State of the Union Address', un grand discours annuel, ça a lieu ici pendant la nuit parce que ils sont $6 \mathrm{~h}$ en retard (...) La BBC et CNN, ils ont fait la 'une' de ça à $6 \mathrm{~h}$ du matin. Bill Clinton dit : 'blablabla'. Nous, nous avons mis ça deuxième mais, pour nous, le lead était la crise croissante en Autriche avec Jorg Haïder qui avait rencontré le 'chancellor' Schüssel la veille (...) On n'est pas forcément européen au point d'être comique mais (...) on essaie toujours de trouver un lead européen et un angle européen sur les histoires, sur les sujets (...) On n'est pas local, mais on n'est pas global (...) C'est un service complémentaire à mon avis aux chaînes nationales ». Les membres historiques de la rédaction semblent d'ailleurs très attachés à cette identité de la chaîne et l'arrivée d'un actionnaire majoritaire britannique ne fut pas forcément vue d'un bon œil. Certains journalistes estiment que l'identité de la chaîne est parfois remise en cause, sa vocation européenne menacée selon eux par la place, jugée grandissante, accordée à l'actualité "anglo-saxonne ». "Ça s'est un peu calmé ces temps-ci mais notamment quand il y avait la guerre au Kosovo, c'était vraiment flagrant, c'est-à-dire que chaque fois que Clinton pétait un coup on avait en direct quoi. Ça c'est un truc qu'on a dû mal à digérer nous parce que... Et... En fait, c'est marrant parce que aussi bien dans les anciens comme moi que dans les nouveaux qui ont très bien...une idée très précise de ce que c'est l'identité européenne, en tous cas de ce qu'ils entendent par là et c'est vrai que a priori Euronews a été créé pour contrebalancer $\mathrm{CNN}$ et Cie, donc pour ne pas avoir une vision anglo-saxonne, enfin c'était le monopole à l'époque et c'était pour faire un contrepoids, avoir une vision européenne. Alors nous, forcément, quand on voit ça, on fait des directs sur Clinton dès qu'il ouvre la bouche, ça énerve, ce n'est pas l'identité de cette chaîne (...) Pendant les événements au Kosovo, c'était flagrant parce que quand il y avait un Américain ou un Anglais qui allait sur place, on faisait des directs patin, couffin et quand le président de l'Union européenne y est allé, on n'a rien fait. On n'a pas fait une news. Alors, c'est pas Euronews ça. C'est ça le problème, c'est que c'est plus Euronews à ce moment-là. » (Entretien, mars 2000) Ce ton distinct de ses concurrents constitue un argument commercial lorsqu'il s'agit de "vendre " la chaîne sur les marchés nationaux auprès des câblo-opérateurs ou des responsables de bouquets satellites. En mettant en évidence le traitement par Euronews d'événements peu ou pas couverts par ses concurrents, notamment en Europe de l'Est, les responsables du service commercial et du service distribution d'Euronews peuvent ainsi souligner l'intérêt que peut représenter la chaîne pour ces téléspectateurs. « CNN a une marque très forte, c'est comme MTV pour reprendre l'équivalent dans un autre 
thème. En Europe, ils sont perçus, ils ont une marque forte, donc tout le monde les connaît. Maintenant, la façon dont Euronews présente les nouvelles est très bien perçue en Europe parce que ce n'est pas américain, parce que notamment en Europe de l'Est, Euronews couvre des sujets qui ne sont jamais couverts, des sujets d'actualité qui ne sont jamais couverts par CNN. Donc nous, forcément, on joue là-dessus aussi. Enfin, quand moi je me présente Euronews, il est bien évident que si mon interlocuteur est pas convaincu et qu'il a besoin d'être convaincu, moi je vais ressortir des arguments sur les couvertures des thèmes d'actualité européens. » (Entretien avec la responsable de la distribution, avril 2000) Cependant, Euronews ne peut ignorer la hiérarchisation et le traitement de l'information de ses concurrents parce qu'elle se situe sur les mêmes marchés mais aussi parce que, comme on l'a vu, toutes les chaînes internationales puisent dans les mêmes sources d'images ou écrites. L' "évidence» de tel ou tel événement est aussi le produit du fait que toutes les chaînes d'information en parlent. Face aux chaînes nationales d'information en continu, Euronews met aussi en avant son point de vue «européen » reprochant à celles-ci d'être précisément trop centrées sur l'actualité nationale. Enfin, les contraintes commerciales s'exerçant sur le contenu éditorial se traduisent dans les conditions de production de l'information. Comme le dit un membre de la rédaction, Euronews fait de la "télé cheap » avec peu de moyens humains (80 journalistes environ) et matériels. Par exemple, le rythme de travail très élevé parce qu'il faut produire l'information de plus en plus vite (réactualisation fréquente des journaux, priorité aux " directs ») ou l'organisation du travail proche de "l'usine » ${ }^{35}$ (« Ici quand on s'assied à la machine à coudre, on coud toute la journée », dit un journaliste) montre à quel point Euronews est aussi un « laboratoire » d'analyse des contraintes économiques qui pèsent sur l'activité journalistique dans les chaînes d'information en continu. L'Europe institutionnelle, un sujet peu médiatique Le traitement de l'Europe institutionnelle illustre bien cette dépendance à l'égard des images et des logiques commerciales, qui peut être en opposition avec les objectifs " européens » de la chaîne. Le type d'images disponibles et leur caractère routinier ne favorisent pas la médiatisation de l'activité des institutions communautaires. La rédaction doit pourtant y consacrer une part importante de ses programmes parce qu'il existe un cadre politique européen, qui plus est en plein développement et donc source d'actualité permanente, et qu'Euronews se veut précisément " européenne ». Le travail des journalistes est donc pris entre des contraintes non seulement éditoriales mais aussi professionnelles et commerciales puisqu'il s'agit de traiter d'un sujet considéré comme «difficile» pour plusieurs raisons. En premier lieu, un présupposé journalistique largement répandu veut que le suivi des activités des institutions européennes se prêterait mal à un traitement médiatique et a fortiori à une couverture télévisuelle. En effet, l'actualité des institutions européennes ne correspond pas aux canons du journalisme de télévision. Elle est considérée comme n'étant absolument pas télégénique (pas «sexy " pour reprendre un terme du jargon journalistique) dans la mesure où les images demeurent très convenues : arrivée des personnalités, bâtiments, tour de table, "shake hands please", sourires, etc. Un chef d'édition d'Euronews explique : «C'est vrai que la Commission et le Parlement on les couvre de plus en plus parce qu'elles ont, ils ont... les institutions européennes ont plus de poids. C'est vrai que le seul problème, c'est toujours les images. Vous avez toujours réunions, conférences de presse. Ça pour la télévision... " ${ }^{36}$. Toutefois, pour des raisons à la fois économiques et techniques, la chaîne a souvent recours aux images et aux interprètes d'Europe by Satellite (EbS). En effet, le service d'information télévisée de l'UE offre l'avantage de 
diffuser des images mais aussi de proposer à ses clients des traductions des discours dans de nombreuses langues, ce qui facilite le travail des journalistes de la rédaction d'Euronews qui n'ont pas alors à traduire et peuvent se contenter de faire une introduction et une conclusion lors des conférences diffusées par EbS. En second lieu, le problème d'images, qui se pose aussi dans la couverture de l'actualité politique par les chaînes nationales, est redoublé par le fait que les contours du système politique européen restent très flous: personnel politique inconnu, absence de symboliques politiques, mécanismes relativement originaux par rapport à la répartition des pouvoirs au niveau national, débats et enjeux tenus pour ésotériques. La conséquence majeure de ce déficit symbolique est la difficulté à mettre en image ce jeu politique. Il n'y a pas de familiarité/ proximité à ce jeu politique qui passe pour de la cuisine interne «bruxello-bruxelloise». D'ailleurs Europa, la rubrique quotidienne d'Euronews, chargée de traiter l'actualité de l'Union européenne, n'échappe pas à cette critique interne d'être trop institutionnelle, trop préoccupée par la vie politique bruxelloise. "C'est chiant cette rubrique, (...) et selon moi, ce n'est pas servir les intérêts de l'Europe parce que c'est vraiment une rubrique très institutionnelle et c'est gonflant à regarder. Moi, je n'ai jamais réussi à la regarder en entier parce que ce n'est pas intéressant (...) Sur l'Europe, on pourrait faire des trucs beaucoup plus vivants, je ne sais pas moi des trucs qui intéressent tout le monde, pas le reflet de ce qui se passe à Bruxelles.» (Entretien avec une journaliste, mars 2000) Ces éléments rendent donc particulièrement laborieuse la couverture de l'actualité des institutions de l'UE. Comme s'en plaignent les journalistes qui couvrent ces questions, il faut ré-expliquer régulièrement ce qu'est le Conseil, le 3eme pilier, etc. Enfin, cette difficulté pose le problème de l'identité de la chaîne, les journalistes d'Euronews cherchant à se défaire d'un traitement trop institutionnel de ces questions ou à se démarquer de l'image de " chaîne de la Commission européenne » que leur renvoient parfois certains confrères par exemple. C'est pour ces raisons à la fois professionnelles et commerciales que les principaux responsables éditoriaux d'Euronews tentent aujourd'hui de traiter des questions européennes autrement, notamment à travers les magazines de coproduction financés par l'UE: Meridian, un mensuel sur les relations extérieures de l'UE et Europeans, un programme hebdomadaire d'actualité "sur tous les grands enjeux européens qui affectent la vie des citoyens ». Il s'agit d'imposer un traitement plus «vivant », qui soit "plus proche des gens » comme le fait remarquer son responsable ${ }^{37}$. Comme dans d'autres domaines traités (l'économie notamment), il s'agit finalement de développer une conception plus commerciale des sujets en insistant moins sur la prise des décisions par exemple que "sur l'impact sur les consommateurs, sur la vie des affaires ${ }^{38}$. A l'instar des autres médias, un autre format éditorial consiste également à rendre compte des activités institutionnelles au travers des "scandales" ou des "polémiques" même si Euronews n'a aucun moyen d'investigation. D'autre part, la part de l'actualité institutionnelle de l'Union européenne a été réduite en temps d'antenne, il y a à la fois moins de « directs » et d'une durée plus courte. «Avant ils se lançaient.. genre le style : quatre heures de débats parlementaires à Strasbourg quoi. Non mais ça c'était des aberrations. Non ça ce genre de truc, on ne fait plus On fait des votes importants à Strasbourg s'il y a lieu ou des discours de... je ne sais pas moi, le président autrichien qui vient se justifier devant les députés où des choses comme ça. Mais on évite les machins soporifiques parce que là c'est... (Entretien avec un rédacteur en chef adjoint, avril 2000) Les contraintes éditoriales : un "point de vue européen " sur l'actualité internationale Une fois ces contraintes définies, il convient également de 
comprendre comment les journalistes d'Euronews construisent (ou " bricolent » ${ }^{39}$ ) très concrètement ce "point de vue européen" forcément flou. L'un des principaux problèmes qu'ils rencontrent tient précisément à l'objet "Europe ", à la traduction éditoriale et journalistique d'un angle voulu " européen ", c'est-à-dire une "télévision porteuse d'une information européenne et d'un regard européen sur le monde " comme l'explique un dépliant publicitaire. Largement vide de sens social, la référence à l'Europe apparaît difficile à expliciter a priori. Interrogés sur le sujet, les enquêtés la définissent d'ailleurs plutôt négativement par ce qu'elle n'est pas : ni anglo-saxonne, ni nationale, ni internationale. Au même titre que d'autres espaces de travail multinational («l'institution-Commission n'est pas la simple juxtaposition des parties qui la composent », notent Marc Abélès et Irène Bellier ${ }^{40}$ ), Euronews tente de définir une identité propre - ce que les journalistes appellent le «ton Euronews »- et qui ne se résume pas à la juxtaposition des points de vue qu'elle héberge. Marc Abélès et Irène Bellier insistent en effet sur la nécessité, pour les agents de la Commission d'inventer des « concepts de compromis », sur le fait que la pratique des fonctionnaires européens n'est pas la somme de ses composantes mais qu'elle est originale dans la mesure où il a fallu inventer de nouvelles méthodes et habitudes adaptées à un contexte multinational où se côtoient des traditions administratives différentes (plurilinguisme qui produit «l'euro-speak », etc.). Ce type de problèmes et d'effets sont aussi le lot de la chaîne et de la même manière que la Commission a dû développer une "culture politique du compromis ", Euronews a dû s'adapter à un contexte où les routines et les traditions professionnelles des journalistes se côtoient et s'entrechoquent au quotidien. $\mathrm{Ni}$ national, ni global Pour expliquer la construction de ce "point de vue européen " difficile à expliciter, les responsables de la chaîne mettent tout d'abord en avant l'objectivité journalistique ( Euronews est porteuse d'un style qui lui est propre dans le traitement des nouvelles, sur lequel ne pèse aucun parti pris politique, national, religieux ou géographique » ${ }^{41}$ ), c'est-à-dire une sorte de point de vue au-dessus des autres points de vue : « Nous, si vous voulez pour un public français, pour nous limiter à la France, on propose un regard un peu au-dessus. On sort de l'hexagone, on se met un peu en altitude... les mauvaises langues vont dire qu'on se met nulle part mais on propose si vous voulez une vision un peu différente par rapport à ce que se fait sur l'écran français d'habitude ", note un rédacteur en chef adjoint de la chaîne. Une nouvelle fois, en l'absence de référents communs, cet angle européen sur l'actualité internationale se définit avant tout par ce qu'il n'est pas. C'est en effet en se démarquant de la concurrence anglo-saxonne ( $\mathrm{CNN}$ et $\mathrm{BBC}$ World) et des chaînes nationales généralistes ou d'information en continu que les journalistes définissent leur approche. C'est ce qu'explique par exemple ce responsable éditorial en prenant l'exemple de la guerre du Kosovo, au cours de laquelle les chaînes nationales, au contraire d'Euronews, se montrèrent souvent extrêmement partisanes en prenant systématiquement selon lui le parti du camp occidental (par la «victimisation » des réfugiés albanais) et en simplifiant à outrance la situation. "On était la télé qui a commencé à s'intéresser la première à ce qui se passait là-bas, avant tous les autres et on a trouvé un langage qui était en contraste total avec ce que faisaient les autres chaînes d'information et y compris les chaînes nationales. Je ne parle pas de la concurrence directe $\mathrm{CNN}$ et $\mathrm{BBC}$, je parle même des chaînes nationales qui étaient, qui se livraient à l'auto complaisance et qui étaient, même si elles avaient des envoyés spéciaux c'était, c'était des lamentations permanentes sur les Albanais et tout ça quoi. Bon, un an après, on a vu qu'on a eu raison d'être un peu sceptique sur ce conflit quoi. 
Donc là on a eu un langage clairement personnel quoi. Un langage clairement personnel sans envoyé spécial, sans équipes de tournage sur place, avec les mêmes images que tout le monde en couvrant les mêmes conférences de presse que tout le monde. » (Entretien avec un rédacteur en chef adjoint, mars 2000) Face à l'absence d'histoire commune, de sens social partagé, les responsables d'Euronews ont dû trouver des parades, des réflexes professionnels leur faisant écarter les sujets qui ne " parleraient » pas à leur(s) public(s) même s'ils peuvent apparaître pertinents à telle ou telle nationalité représentée au sein de la chaîne. «Et donc, ce qu'on a essayé de faire déjà c'est d'avoir une cohérence éditoriale. Elle est apparue en fait assez spontanément (...) parce que Euronews souffre des maux dont souffre l'Europe quoi. Ça a les avantages et les inconvénients de l'Europe vu que nous en sommes en partie l'émanation... spirituelle je veux dire (rires) (...) Il n'y a pas franchement de dénominateur commun aux intérêts de tous les européens et tout ça quoi, donc éditorialement c'est assez difficile de construire. Alors moi je suis allé, je suis parti du principe que, pour nous limiter à l'Europe, ce qui peut intéresser un Européen c'est un sujet qui affecte, par exemple un sujet allemand qui peut affecter tous les européens. (...) Ou alors mettons ce qui peut surprendre chez un voisin.» (Entretien avec un rédacteur en chef adjoint, mars 2000) Ce «bricolage " se manifeste par exemple dans les principes généraux qui guident la sélection des sujets : un sujet national ayant des répercussions dans les autres pays membres, un sujet pouvant susciter la surprise par l'anecdote (ce qui peut «surprendre " dans les comportements des pays voisins ${ }^{42}$ ) ou encore un sujet international traité sous un angle mettant en évidence les connexions européennes d'événements se produisant hors de l'Europe géographique. Une autre réponse trouvée par la rédaction est incarnée par le magazine Perspectives qui propose une sélection de reportages des chaînes nationales partenaires sur un sujet identique. L'objectif affiché est ici de donner aux téléspectateurs la possibilité de voir comment l'information est perçue et traitée dans les pays européens à travers une reprise des principaux sujets des journaux télévisés du soir du Royaume-Uni, de France, d'Allemagne, d'Espagne, de Suisse et d'Italie. Ici, l'Europe est construite au pluriel, par la juxtaposition de points de vue journalistiques nationaux et non par la fabrication d'un hypothétique point de vue européen. La journaliste responsable de ce magazine rappelle d'ailleurs que ce type d'approche interdit pratiquement un certain nombre de sujets qui, s'ils sont pertinents pour Euronews, ne sont pas traités par toutes les chaînes nationales. " D'abord, on choisit un thème et c'est pas forcément mon choix dans la mesure où ça dépend de ce qui a été fait. Les sujets, je ne peux pas les inventer. Parfois, c'est vrai que...je me souviens quand il y a eu par exemple la signature de l'accord en Irlande du Nord. Moi, je voulais faire l'Irlande du Nord mais les autres, les chaînes nationales, ça c'est typiquement le sujet dont ils s'en foutent royalement (rires) (...) A chaque fois que j'ai voulu faire l'Irlande du Nord, ce n'est pas possible puisqu'ils n'envoient pas de correspondants, ils font des sujets un peu 'merdouilles' (?), enfin factuels et le choix il se fait sur un truc où il $\mathrm{y}$ a des correspondants sur place, où ils font des reportages vraiment. Parce que si c'est pour revoir les mêmes images que dans les agences, ça ne vaut pas le coup. Il faut des sujets 'anglés'.» (Entretien avec une journaliste, mars 2000) Ce "point de vue européen" se construit aussi dans les commentaires rédigés par les journalistes. Ils se doivent d'éviter toute référence à un contexte national particulier car même s'ils sont des journalistes de «langue », ils ne s'adressent pas à un pays en particulier (hormis le cas des Italiens). Par exemple, un journaliste français (francophone serait d'ailleurs plus approprié) ne s'adresse pas 
uniquement aux téléspectateurs de son pays mais aussi à des Québécois, des Suisses, des Belges, des Africains, des Maghrébins et plus largement à des francophones. Le cas le plus emblématique est sans doute celui des journalistes anglais dans la mesure où Euronews n'est pas ou peu diffusé en Grande-Bretagne. De facto, ces journalistes ne s'adressent donc pas seulement à leurs compatriotes. - « On n'écrit pas pour un public britannique, donc il faut toujours penser qu'on écrit pour les Scandinaves, pour les gens qui sont dans le bassin méditerranéen, même dans l'Europe de l'Est, on ne sait pas vraiment forcément où les gens sont, nous écoutent en anglais. Ça, ça change des choses, des façons dont on écrit, les termes qu'on utilise... - C'est-à-dire des termes beaucoup plus simples. - Oui, il faut éviter de faire trop de jeux de mots, il faut une clarté de style et, pour les Français aussi, ils pensent qu'ils écrivent que pour les Français mais, en fait, ils écrivent pour les francophones belges, pour les francophones suisses et au Canada, c'est diffusé au Canada. Peut-être qu'ils ont un peu tendance d'oublier qu'ils écrivent pour ce public-là mais... Dès le début, moi, j'étais très consciente que c'est pas regardé du tout en Angleterre, au Royaume Uni alors vraiment pour les anglophones soi-disant ou les gens qui avaient anglais comme leur deuxième langue ailleurs, en Grèce par exemple (...) Et les Espagnols, ils écrivent... Maintenant, ils écrivent pour l'Amérique du Sud, ils sont diffusés là-bas, et les Italiens, ils écrivent pour leur pays (...) et peut-être en Albanie (...) Les Allemands, c'est Autriche, c'est la Suisse Romande. Oui, les Italiens, c'est écouté en Suisse.» (Entretien avec une journaliste anglaise, avril 2000) L'existence de débats permanents au sein de la rédaction montre la difficulté de trouver une cohérence éditoriale qui satisfasse l'ensemble des journalistes de la chaîne. Les pratiques journalistiques, qui tiennent parfois aux législations ou aux habitudes nationales, varient quand il s'agit de montrer un visage d'un mineur ou encore des seins nus à l'antenne. Comment doit se comporter Euronews quand des télévisions nationales choisissent de montrer le visage d'un enfant accusé dans une affaire tandis que d'autres préfèrent mettre une mosaïque sur son visage? Finalement, la rédaction en chef optera de le faire apparaître à l'écran, choisissant la législation la moins contraignante. Dans le second cas, les journalistes responsables des montages ont dû renoncer à montrer des poitrines féminines filmées lors du Carnaval de Rio car le directeur de la chaîne, un journaliste écossais, y était opposé, imposant une pratique en cours au Royaume Uni. L'importance du montage Dans la mesure où Euronews diffuse en six langues, l'organisation de l'entreprise et les pratiques professionnelles doivent s'ajuster de façon à ce que l'information diffusée soit sensiblement la même, quel que soit le journaliste en charge du commentaire. Ce problème de la cohérence de l'antenne renvoie en fait à un double processus: d'une part, les responsables de la sélection et du montage des sujets doivent s'assurer que les divers commentaires nationaux puissent s'adapter à un canevas commun; d'autre part, les journalistes de langue ne doivent pas perdre de vue que leur public est multinational et qu'en conséquence ils ne peuvent mobiliser les références nationales traditionnelles. Si la chaîne ne fabrique pas un produit uniforme puisque chaque journaliste écrit lui-même son commentaire, il a fallu faire en sorte d'assurer une forme cohérente à l'antenne. En effet, on ne trouve pas au sein de la rédaction d'Euronews autant de rédactions que de langues diffusées mais une hiérarchie commune et des journalistes de langue rattachés à chaque rubrique et édition. Afin de voir au concret comment Euronews produit cette cohérence, il semble intéressant de suivre le déroulement de la chaîne de production de l'information. C'est là en effet qu'est mis à l'épreuve le concept d'information européenne, que l'organisation de la chaîne et les procédés pratiques sont mis en 
œuvre. Une fois les décisions prises concernant les sujets devant être réalisés, ce sont les chefs d'édition et de rubriques qui tiennent une place déterminante dans la production d'Euronews. La chaîne comprend trois chefs de rubrique (Sport, Economia, Europa) et six chefs d'édition (chacun étant chargé d'assurer une partie des 20 heures quotidienne d'antenne). Ils assurent le montage des sujets à partir des images que le service de coordination news ${ }^{43}$ leur a fourni. Lorsqu'ils montent leur sujet, les chefs d'édition et de rubriques doivent garder en tête l'idée que les six langues doivent pouvoir s'adapter aux images. Ils assistent à la conférence de rédaction du matin, sélectionnent les sujets du jour et en réalisent le montage si les images sont déjà disponibles. Ils montrent et expliquent aux six journalistes « de langue » qui assureront le commentaire, le sujet et le montage qu'ils ont choisis. Une discussion sur le reportage, sa pertinence, le commentaire devant l'accompagner peut alors prendre place. Selon les nationalités, les traditions professionnelles ${ }^{44}$, les sensibilités à l'égard d'un sujet peuvent s'avérer différentes : certains en contestent l'intérêt pour "son " public, expliquant que le montage réalisé n'est pas adapté à la structure de la langue ou aux habitudes télévisuelles de son pays. D'ailleurs, les chefs d'édition tranchent assez vite les débats et il n'y a guère de place pour la discussion dans la mesure où ces journalistes disposent parfois seulement de quinze minutes pour réaliser leur mixage : écrire leur commentaire à partir des dépêches d'agences disponibles ou de la documentation fournie par le service de prévision ${ }^{45}$ et entrer en cabine de mixage pour réaliser le son du sujet. Ce sont là des conditions extrêmes de production de l'actualité quand on les compare à celles existant dans les chaînes nationales. Autrement dit, les montages sont relativement routiniers. Par exemple, un chef d'édition de la chaîne devait faire un montage de 45 secondes d'une rencontre diffusée en partie en direct à la mi-journée entre Thomas Klestil, le président autrichien, et Romano Prodi, le président de la Commission européenne. Avant même que ne commence la conférence commune, il a déjà prévu de mettre en tête ( 15 secondes) Thomas Klestil autour d'une table (« avec ses 14 copains ", rigole un chef d'édition), puis l'arrivée des deux personnalités dans les couloirs et dans la salle. Entre la quinzième et la trente quatrième seconde, un ou deux extraits des discours pour finir (de 34 à 45) sur le shake hand dans une petite salle connexe à la salle de conférence. Cependant, c'est le rôle des chefs d'édition et de rubriques que d'anticiper les problèmes (sujet et angle non national, structure langagière) et de réaliser un montage "passe-partout", adaptable au différentes situations ${ }^{46}$. "Je cherche un peu à faire la synthèse de la manière dont les six journalistes peuvent écrire le sujet (...) C'est un peu comme un légo, chacun peut écrire en fonction de ça ", indique un chef de rubrique ${ }^{47}$. C'est dans ce montage, qui constitue un compromis entre les différentes traditions journalistiques nationales, que se réalise le « ton Euronews ». « C'est ce que je dis quand il y a des chefs d'ed (ndt : chef d'édition) nouveaux qui commencent ou des remplaçants : 'tu arrêtes de réfléchir dans ta langue, tu réfléchis dans toutes les autres langues. Et tu réfléchis pas dans ta langue en construisant un sujet. Tu construis le sujet en réfléchissant dans d'autres langues pour voir comment les autres peuvent l'écrire'.(...) Il faut trouver le langage des images commun, le langage des explications données par le chef d'ed commun, la structure commune à tout ça pour arriver à un discours qui se tienne entre certains paramètres quoi. Ce n'est peut-être pas copié, identique en toutes les langues mais enfin que le message soit le même quoi. » (Entretien avec un rédacteur en chef adjoint, avril 2000) Les contraintes organisationnelles Euronews présente comme on l'a vu plusieurs spécificités liées à son caractère multilingue. C'est d'abord une chaîne sans 
présentateur et sans plateaux, c'est-à-dire une télévision sans visage qui diffuse des images en continu et dans des formats relativement courts. Il serait dans ces conditions extrêmement coûteux pour la chaîne de mettre en place plateaux et présentateurs correspondants à chaque langue de diffusion. La déclinaison en six langues impose également une organisation assez lourde en équipes de six journalistes à chaque sujet diffusé. Les difficultés à constituer un média dit européen se traduisent plus encore dans l'organisation du personnel - ou tout du moins des journalistes (la plupart des administratifs et des techniciens sont recrutés en France) - car elle se heurte aux différences entre les marchés du travail nationaux. Mais cette organisation présente des spécificités qui tiennent tout d'abord aux conditions de production, tout particulièrement au rythme de travail auquel sont soumis journalistes. C'est ce qui explique la moyenne d'âge, située en dessous de 30 ans selon de nombreux interlocuteurs, la rotation des personnels relativement élevée, par rapport aux chaînes hertziennes nationales. Ces particularités, qui sont aussi celles de nombreuses nouvelles chaînes diffusées par le câble et le satellite ${ }^{48}$, ont des effets sur le recrutement des jeunes journalistes et leur gestion compte tenu des faibles possibilités de carrière. « Il y a un turn-over très fort, il y a beaucoup de gens qui arrivent et qui n'ont pas forcément beaucoup travaillé avant d'arriver ici, pour lesquels je pense que c'est un excellent tremplin. Mais le problème, c'est que ça reste trop souvent un tremplin. On a une machine qui tourne, qui est un peu une usine d'infos mais, au bout d'un moment, on stagne en termes de 'carrière' soit on passe par les étapes : chef d'édition, chef de rubrique, producer etc. Mais il n'y a pas 36 places hein...» (Entretien avec une journaliste producer, avril 2000) Une grande partie du recrutement et de la gestion des équipes de journalistes est liée à la spécificité européenne d'Euronews qui constitue une sorte de révélateur miniature des variations nationales des espaces du journalisme ${ }^{49}$. En effet, les équipes espagnoles et italiennes ${ }^{50}$ ne posent pas de gros problème de recrutement car elles trouvent à Euronews une situation financière bien meilleure que dans leur pays d'origine où le travail est plus rare et les salaires généralement moins élevés qu'en France. Ces équipes tendent donc à être composées de journalistes plus âgés, plus stables au sens où une partie d'entre eux s'est installée depuis plusieurs années dans la région lyonnaise. A l'inverse, les équipes de langues anglaise et allemande sont plus difficiles à gérer car le marché de l'emploi journalistique en Allemagne comme en Angleterre offre de nombreuses opportunités. Le recrutement des jeunes journalistes se révèle plus délicat et ils ne restent pas très longtemps à leurs postes. En effet, les jeunes journalistes britanniques semblent envisager dans leur majorité le travail à Euronews comme une expérience très "formatrice » qui permet à certains de revenir dans le pays d'origine au sein des chaînes nationales ou internationales d'information en continu, ou encore d'intégrer des agences de presse audiovisuelles dont les sièges sont à Londres. L'arrivée d'ITN comme actionnaire majoritaire a également facilité le recrutement de journalistes anglais qui, compte tenu de la faible notoriété d'Euronews au Royaume-Uni, espèrent bénéficier de l'effet de notoriété de cette société. «Pour les Anglais, la source la plus grande sûre c'est la radio locale : les journalistes sont jeunes, ils ne sont pas trop bien payés, trop bien salariés et, pour eux, surtout quand on est jeune, c'est toujours une grande aventure de venir en France de venir apprendre une autre langue, d'apprendre, si on n'a traité uniquement l'actualité locale, de venir ici et de traiter tout le monde (...) Et aussi il y a la question du direct. Comme je vous l'ai dit, il y a des directs faits comme ça avec dix minutes de préavis et commentaire en direct pour des grands événements 
et c'est une très bonne formation ça pour les chaînes d'infos maintenant où tout le monde est obligé de faire du direct tout le temps. Et bon pour les journalistes, il faut trouver des jeunes qui comprennent les avantages d'être ici pour un an ou deux ans mais on ne peut jamais attendre qu'ils vont rester plus que ça.» (Entretien avec le directeur de la rédaction d'Euronews, de nationalité écossaise, mars 2000) La situation des journalistes français se situe dans l'entre-deux à la fois dans le recrutement et la rotation des personnels. Les responsables s'appuient sur le marché national et local pour recruter des pigistes et/ou des titulaires. L'un des rédacteurs en chef explique ainsi ses difficultés à trouver des jeunes journalistes "opérationnels " rapidement et dotés d'une « culture internationale » suffisante. Les journalistes français peuvent donc être en poste depuis plusieurs années alors que d'autres ne font qu'y passer. Dans tous les cas, il est difficile de trouver à la fois des jeunes journalistes avec quelques années d'expérience dans le métier dotés d'une "culture internationale » et qui de surcroît acceptent un poste de journaliste news. Les différences nationales se font également sentir dans les rémunérations où l'harmonisation européenne n'existe pas. Afin d'attirer de nouvelles recrues, la direction des ressources humaines a donc offert des rémunérations plus intéressantes aux journalistes provenant de pays où les marchés du travail journalistique sont les plus ouverts (Allemagne et Royaume-Uni). Ces quelques éléments permettent donc d'entrevoir les problèmes extrêmement concrets que suppose le fonctionnement d'une chaîne abritant six nationalités. Des journalistes ajustés Pour adapter les commentaires à chacune des six langues, les nouveaux journalistes apprennent sur le tas à éviter de mobiliser des références explicitement nationales ou des expressions trop idiomatiques qui ne seraient pas forcément comprises en dehors de leur pays d'origine ${ }^{51}$. Ils doivent donc, dans le cas où ils ont des expériences journalistiques nationales, «désapprendre " un certain nombre de leurs habitudes et routines professionnelles. "Je veux dire, chaque fois qu'un journaliste français commence ici en piges ou en contrat ou n'importe quoi, la première chose que je lui dis... euh 'oublie, oublie que t'es français, oublie que tu es en France, tu travailles pas pour une télévision française. Il se trouve que tu parles français, que tu vas travailler en français, mais c'est tout. Tu t'adresses à un public qui dépasse largement la France' (...) Donc 'sors de ta peau de Français, tu fais pas une télé française. OK ?'.» (Entretien avec un rédacteur en chef adjoint, avril 2000). Les jeunes journalistes présentent dans ces conditions des profils ajustés aux besoins d'Euronews. Jugés plus malléables, ils seront, d'après les responsables éditoriaux, plus à même d'intégrer les habitudes de la chaîne. Après quelques jours passés assistés par un journaliste plus ancien, leurs premiers sujets sont surveillés de près et les chefs d'édition leur font remixer leurs commentaires jusqu'à ce qu'ils soient jugés acceptables. Il existe par ailleurs un système de relecture entre journalistes d'une même langue et selon les compétences linguistiques de chacun, sorte de contrôle croisé permettant de corriger d'éventuelles erreurs. Enfin, l'étude des trajectoires sociales et professionnelles permet de dégager quelques caractéristiques des journalistes d'Euronews, qui tiennent au moins en partie à sa dimension "européenne ». Au-delà de leurs expériences multimédias et bien évidemment de leur maîtrise de deux ou trois langues au minimum, de nombreux journalistes - c'est particulièrement vrai pour les plus anciens qui avaient dû se soumettre à un recrutement très sélectif ${ }^{52}$ - ont non seulement des expériences à l'étranger mais ont travaillé pour des médias d'une langue différente de celle de leur pays d'origine. Comme le dit un rédacteur en chef adjoint en parlant des journalistes embauchés lors des premières années de diffusion de la chaîne, « c'étaient 
des gens qui étaient rarement au-dessous de trente ans et c'étaient des gens qui avaient tous une expérience à l'étranger, une expérience nationale et une expérience dans un autre pays que celui d'origine ${ }^{53}$. Ce n'est pas forcément le cas des jeunes journalistes recrutés ces dernières années pour lesquels l'expérience est nouvelle. Ce profil international se dégage dans de nombreux cursus scolaires des jeunes journalistes qui ont pour partie suivi des études à l'étranger dans le cadre du développement des programmes et des échanges entre les universités européennes ou ont suivi des formations en relations internationales. D'autres ont vécu pendant plusieurs années à l'étranger pour le travail ou simplement parce qu'ils ont suivi leurs parents. Ce «bricolage » quotidien d'un " point de vue européen » sur l'information internationale, c'est-à-dire aussi la recherche d'une cohérence éditoriale, doit beaucoup à quelques «anciens» qui occupent pour la plupart des postes de responsabilité. Ils essaient d'imposer ce «ton Euronews » aux jeunes journalistes. En fait, celui-ci est le produit à la fois d'une conception élevée de l'information, faisant la part belle aux sujets internationaux peu traités par les chaînes nationales, et des exigences commerciales de plus en plus fortes. Les journalistes sont constamment partagés entre les objectifs "européens" de la chaîne (promouvoir une identité européenne, défendre une conception de l'information différente des anglo-saxons, etc.) et les contraintes multiples évoquées plus haut. C'est ce qui explique que le contenu même de ce qui est appelé l' « information internationale » ou " européenne » a considérablement changé comme dans les autres types de médias. Ce type d'actualité s'est considérablement diversifié. Elle recouvre moins aujourd'hui une information strictement institutionnelle (notamment diplomatique) qu'une information soit traitée sous forme de faits divers (les guerres, les catastrophes, les accidents, les risques, les scandales, etc.), c'est-à-dire des hard news, soit à travers des exemples pouvant susciter des identifications multiples de la part des téléspectateurs (histoires « humaines», sujets pratiques, etc.), soit parce qu'elle repose sur des «belles images » pour reprendre une expression souvent employée par les journalistes de la chaîne.

2 Annexe méthodologique Cette enquête ${ }^{54} s^{\prime}$ 'appuie sur une vingtaine d'entretiens avec les personnels de la chaîne (différents types de journalistes, responsables des ressources humaines, de la distribution, des relations avec les téléspectateurs, directeur artistique, etc.) et de nombreuses observations ethnographiques réalisées lors de deux séjours de deux jours au siège de la chaîne à Ecully dans la banlieue lyonnaise. Durant ces périodes, la direction de la chaîne ${ }^{55}$ nous a permis de circuler dans la rédaction, de faire des entretiens et d'assister aux conférences de rédaction. Cette enquête a également été complétée par plusieurs interviews auprès d'anciens journalistes de la chaîne mais aussi de personnes travaillant au siège de l'Union européenne de radio-télévision $\left(U_{E R}{ }^{56}\right)$ à Genève ${ }^{57}$ où se réalisent les Euro Vision News (EVN), des échanges d'images entre de nombreuses chaînes européennes. Nous avons aussi été autorisés pendant deux jours à suivre le travail de la coordination news de l'UER ${ }^{58}$. Ce complément était indispensable pour comprendre en amont comment sont produites et sélectionnées les images qui constituent une des principales sources pour les journalistes d'Euronews. Bien évidemment, ce travail s'est accompagné de recherches documentaires (net, revues de presse, annuaires biographiques, statistiques d'audience, articles de revues et ouvrages essentiellement) sur la chaîne mais, plus largement, sur la production des news à l'échelle internationale et les médias en Europe ou encore sur le marché des chaînes du câble et du satellite. Il a également donné lieu à de nombreux enregistrements et visionnages des différents programmes d'Euronews à des périodes 
s'étalant du mois de janvier 1999 au mois d'août 2000. Si nous n'avons pas utilisé certains outils statistiques ou des méthodes d'analyses de contenu (qui auraient pu consister à comparer par exemple les différentes versions des commentaires de sujets diffusés par Euronews), c'est parce que nous avons voulu mettre l'accent sur les contraintes de la production journalistique et leurs effets sur l'information transmise par une chaîne à vocation européenne.

\section{NOTES}

1. Une autre recherche en cours est menée par Olivier Baisnée sur un autre « laboratoire » du journalisme en Europe, celui des journalistes qui couvrent « l'actualité européenne » à Bruxelles et à Strasbourg

2. Extrait d'un document de présentation de la chaîne, 2000.

3. Parmi les rares travaux sur le sujet, on renvoie à la thèse de Jean-Michel Utard (Arte : information télévisée et construction d'un point de vue transnational. Etude d'un corpus franco-allemand, Strasbourg, thèse pour le doctorat en Sciences de l'information et de la communication sous la direction d'Yves Lavoinne, 1997) et à l'article de Marcel Machill (« Euronews : the first European news channel as a case study for media industry development in Europe and for spectra of transnational journalism research ", Media, Culture and Society, vol 20, n³, 1998, pp. 427-450). 4. Mais ce terrain d'enquête s'inscrit plus largement dans une recherche à long terme qui se propose d'analyser les conditions de production de l'information dans différents domaines de l'activité journalistique : médecine, science, justice, politique, sportif, intellectuel, etc. C'était donc l'occasion d'étudier ce que les journalistes appellent l'information « internationale » ou « l'étranger » telle qu'elle peut être produite dans les chaînes de télévision, tout particulièrement les chaînes d'information en continu qui se sont développées dans les années 80 et 90 . Euronews est aussi un terrain d'étude privilégié des transformations des conditions de production de l'activité journalistique à la télévision depuis le début des années 80 .

5. Les analyses en termes de champ, c'est-à-dire en termes relationnels, ont rarement été mises en œuvre à une échelle internationale. Pour des illustrations publiées en France, voir notamment : Pierre Bourdieu et Loïc Wacquant, «Sur les ruses de la raison impérialiste ", Actes de la recherche en sciences sociales, n¹21-122, 1998, p.109-118 ; Pascale Casanova, La République mondiale des Lettres, Paris, Seuil, 1999.

6. Helen Kelly-Holmes, European Television Discourse in Transition, Clevedon, Multilingual Matters LTD, 1999.

7. A titre d'exemples, on peut citer les cas d'Eurosport International diffusée en 16 langues en 2000 et qui compte deux versions nationales en France et au Royaume Uni, de MTV et de CNN qui ont développé d'autres langues que la langue anglaise (CNN en espagnol en 1997), voire des programmes spécifiquement nationaux (par exemple MTV Italy, MTV UK).

8. Jean-Michel Utard, Arte : information télévisée et construction d'un point de vue transnational. Etude d'un corpus franco-allemand, Strasbourg, op. cit., p. 58. 
9. Alex Taylor, « The Europeanization of Programming » in Michael Scriven et Monia Lecomte (eds), Television Broadcasting in Contemporary France and Britain, New-York, Oxford, Berghahn Books, 1999, pp. 168-174.

10. Richard Collins, «The European Union Audiovisual Policies of the U.K. and France » in Michael Scriven et Monia Lecomte (eds), Television Broadcasting in Contemporary France and Britain, op. cit., p. 200.

11. Richard Collins, "Supper with the devil - a case study in private/public collaboration in broadcasting : the genesis of Eurosport ", Media, Culture and Society, vol 20, n4, p. 655 face au développement des chaînes commerciales[[Le Monde, 4 décembre 1993.

12. Préface au livre de Akiba A. Cohen, Mark R. Levy, Itzhak Roeh, Michael Gurevitch, Global Newsrooms, Local Audiences. A Study of the Eurovision News Exchange, Londres, John Libbey, 1996, p. xii.

13. Si les télévisions allemandes (ZDF et ARD) et anglaise (BBC) figurent parmi les principaux membres actifs de l'UER, elles n'ont pas participé à la création d'Euronews à l'inverse des chaînes françaises (France 2, France 3), italienne (RAI), chypriote (cyBC), grecque (ERT), égyptienne (ERTU), belge (RTBF), portugaise (RTP), espagnole (TVE), monégasque (TMC) et finlandaise (YLE).

14. Marcel Machill, «Euronews : the first European news channel as a case study for media industry development in Europe and for spectra of transnational journalism research », art. cit., pp. 428-429.

15. Chris Shore (Building Europe, London, Routledge, 2000, p. 258) rappelle ainsi que, dès 1985, le rapport Adonnino (Pietro Adonnino, «A People's Europe : Reports from the Ad Hoc Committee », Bulletin of the European Communities, supplement 7/85, Luxembourg, 1985) commandé en 1984 par le Conseil européen à la suite de la faible participation aux élections européennes suggérait, parmi d'autres mesures visant à « renforcer et promouvoir l'identité de la Communauté et son image à la fois pour ses citoyens et pour le reste du monde », que soit créée une " aire audiovisuelle» européenne passant notamment par la mise en place d'une chaîne télévisée plurilinguistique "véritablement européenne ».

16. Commission of the European Communities, Television without Frontiers : Green Paper on the Establishment of the Common Market for Broadcasting, Especially by Satellite and Cable, Brussels, 1984 cité par Philip Schlesinger, Media, State and Nation, Sage, 1991, p. 139.

17. Commission of the European Communities, The European Community and Culture, Brussels, 1985 cité par Philip Schlesinger, op. cit., p. 139.

18. Philip Schlesinger, op. cit.

19. Pietro Adonnino, "A People's Europe : Reports from the Ad Hoc Committee”, Bulletin of the European Communities, supplement 7/85, Luxembourg, 1985 cité par Chris Shore, Building Europe, Routledge, 2000.

20. Peter Pex, Rapport sur la politique d'information et de communication dans l'Union européenne, A4-0115/98, 32 p., 5 mai 1998.

21. Conception dont se plaint d'ailleurs une journaliste d'Euronews qui assure une veille informationnelle à Bruxelles en indiquant que lorsqu'elle refuse à un parlementaire de le filmer « souvent les parlementaires pensent que ce n'est pas correct, ils veulent être toujours à la télévision » car le financement européen dont bénéficie Euronews, leur ouvrirait un droit à l'antenne.

22. Entretien, mars 2000. 
23. Chris Shore, Building Europe, op. cit., p.51.

24. Philip Schlesinger, «From cultural defence to political culture : media, politics and collective identity in the European Union », Media, Culture and society, 1997, Vol. 19, pp. 369-391.

25. Seuls certains magazines financés par la Commission européenne utilisent des images tournées pour la chaîne.

26. Entretien, avril 2000.

27. Dans un travail comparatif sur plusieurs journaux télévisés dans différents pays, Eliséo Veron avait montré comment ils s'inscrivaient dans des cadres nationaux : « Il est là, je le vois, il me parle », Communications, n³8, 1993.

28. Olivier Boyd-Barret et Michel Palmer, Le trafic des nouvelles, Paris, Moreau, 1981, pp. 652-653.

29. Entretien, mars 2000.

30. Les services de base correspondent aux offres les moins chères proposées par les responsables des bouquets satellites et du câble.

31. Les revenus de la chaîne proviennent de la vente d'espaces publicitaires, de contrats de parrainage, des abonnements souscrits par les câblo-opérateurs, des droits des diffuseurs hertziens qui retransmettent ses programmes. D'autres revenus sont générés par la vente de ses propres productions et par des contrats de coproduction passés avec l'Union Européenne.

32. Pour effectuer une analyse détaillée, il faudrait établir des comparaisons entre les situations très variables des marchés du câble et du satellite et l'intérêt forcément très différent qu'y accorde le chaîne. En effet, comme dans le cas d'Eurosport International, l'Allemagne représente le marché où le potentiel d'audience et de publicité est le plus grand.

33. Sur ce point, on peut se reporter utilement à l'étude de François Heinderycks : L'Europe des médias, op. cit., chapitre 2.

34. Entretien, mars 2000.

35. Expression employée par un membre de la rédaction lors d'un entretien réalisé en mars 2000.

36. Entretien, mars 2000.

37. Les termes sont extraits d'un entretien avec le rédacteur en chef adjoint, chargé des magazines, mars 2000.

38. Brochure du service communication de la chaîne, 1998.

39. Le verbe bricoler et le terme bricolage ne sont pas utilisés dans un sens péjoratif. Ils visent simplement à spécifier comment les journalistes tentent de fabriquer au sens artisanal une cohérence éditoriale.

40. Marc Abélès et Irène Bellier, « La Commission européenne, du compromis culturel à la culture politique du compromis ", Revue Française de Science Politique, vol. 46, n³ juin 1996, p. 434.

41. Brochure du service communication de la chaîne, 1998.

42. On voit bien ici que, confrontés à une identité européenne introuvable et derrière un discours qui idéalise le point de vue 'européen', la réactivation des stéréotypes nationaux est une des « recettes » qui « marchent » pour produire des sujets qui " parlent » aux téléspectateurs européens.

43. Le service de coordination est le nœud informationnel de la chaîne, la « tour de contrôle » comme l'explique l'un de ses membres. C'est en effet dans cette petite pièce qu'arrivent les images en provenance des chaînes partenaires, d'ITN, des EVN et des 
agences audiovisuelles. Face à un mur d'images, les membres de la coordination réalisent des scripts décrivant les sujets disponibles (qui sont accessibles à tous les membres de la rédaction à partir d'une base de données), reçoivent et sélectionnent les images correspondant aux sujets souhaités par la rédaction.

44. Au cours des entretiens sont revenus, souvent avec ironie, des remarques sur le lyrisme et le côté désordonné des montages méditerranéens auxquels s'oppose celui des allemands décrit comme gage de sérieux et de cohérence.

45. Ce service a pour charge de réaliser une veille informationnelle, d'anticiper les événements à venir, notamment les directs, afin que la rédaction ne se trouve pas prise au dépourvu. C'est également ce service qui réunit la documentation (Internet, dépêches, coupures de presse) relative aux sujets à venir ; documentation que les journalistes pourront consulter à partir d'une base de données informatisée afin de préparer à l'avance la rédaction des commentaires qu'ils enchaînent à un rythme très élevé. Cette documentation se révèle également indispensable pour la réalisation des directs afin que les journalistes puissent contextualiser et commenter des images qu'ils découvrent au moment de la prise d'antenne.

46. Une nouvelle fois le parallèle avec ce qu'écrivent Abélès et Bellier (op. cit., p. 432) à propos des agents de la Commission européenne peut être établi lorsqu'ils remarquent que « dans le travail, chacun côtoie quotidiennement des fonctionnaires d'origine différente. Cette diversité des langues et des cultures n'est pas sans conséquences sur les manières de penser et d'agir et sur les productions de la Commission » et que « se fabrique dans l'espace de la Commission européenne 'un compromis culturel' par lequel se combinent et se recomposent des appartenances et des identités très diverses au nom d'un projet commun. Cela engendre dans le champ d'action de cette institution une 'culture politique du compromis' marquée par des orientations et des concepts qui conjuguent plus ou moins heureusement des traditions parfois très différentes ».

47. Entretien, avril 2000.

48. Des entretiens menés à Eurosport International, Eurosport France et à L'Equipe TV le confirment.

49. Comprendre complètement les variations nationales sur les marchés du travail journalistique impliquerait d'analyser les systèmes de formation et la durée moyenne des études des étudiants qui deviennent journalistes. C'est ainsi par exemple qu'il y a des différences tenant à l'âge d'entrée dans la profession. Les Anglais et les Espagnols suivent des formations courtes alors que les Allemands et les Italiens accèdent au journalisme après des cursus plus longs et donc plutôt après $25 / 26$ ans. Une telle étude justifierait à elle seule une recherche à part entière qui n'est pas l'objet du présent article.

50. Il est difficile d'avoir des observations précises dans le cas des équipes de langue portugaise dans la mesure où le Portugais a été introduit quelques mois avant le début de notre enquête.

51. Dans son livre sur le Parlement européen, Marc Abélès (Marc Abélès, La vie quotidienne au Parlement européen, Hachette, 1992, p. 364 et suiv.) insiste sur les difficultés qu'implique la communication politique en direction d'un auditoire multinational. Les formes traditionnelles du discours politique (humour, rappels historiques) doivent dans ces conditions être proscrites car elles seront difficilement comprises par les destinataires.

52. Le Monde, 6 janvier 1993.

53. Entretien, mars 2000. 
54. Cette enquête coordonnée par le Centre de recherches administratives et politiques (CRAP) a été financée par le programme « Identité européenne » du CNRS.

55. Nous tenons à remercier l'ensemble des journalistes et plus généralement des personnels de la chaîne, tout particulièrement Dominique Gicquel, responsable des ressources humaines, qui a contribué à convaincre sa hiérarchie de l'intérêt d'une telle étude, et Bill Dunlop, le directeur de la rédaction, de nous avoir permis de réaliser un tel travail.

56. L'UER correspond à une traduction du sigle anglais European Broadcasting Union (EBU) plus utilisé.

57. Nous remercions les différents personnels de l'UER qui nous ont accordé un peu de leur temps, notamment Adina Fulga qui a beaucoup contribué à nous faciliter l'accès à la coordination news.

58. Ce travail a été réalisé par Jean Chalaby (City University of London), Eric Darras (IEP Toulouse) et Dominique Marchetti (CRAP, CNRS).

INDEX

Index géographique : Europe

Mots-clés : construction européenne, médias 